\title{
COMPARAÇÃO DO PROCESSO DE ADSORÇÃO DO CORANTE REATIVO PRETO 5 UTILIZANDO CARVÃO COMERCIAL DE CASCA DE COCO E LODO ATIVADO GASEIFICADO
}

\author{
R. F. dos SANTOS ${ }^{1}$, J. J. SORNAS ${ }^{1}$, W. UTECH JR ${ }^{1}$, A. R. VASQUES ${ }^{2}$, R. C. S. C. \\ VALLE $^{1}$, E. FONTANA ${ }^{1}$, J. A. B. VALLE ${ }^{1}$, C. MARANGONI ${ }^{1}$ e C. R. L. AGUIAR ${ }^{1}$ \\ ${ }^{1}$ Universidade Federal de Santa Catarina, Campus Blumenau, Curso de Engenharia Têxtil \\ ${ }^{2}$ Universidade de Brasília, Instituto de Química \\ E-mail para contato: renan.felinto93@gmail.com
}

\begin{abstract}
RESUMO - A adsorção do corante reativo preto 5 foi avaliada testando-se o carvão comercial de casca de coco e um obtido a partir da gaseificação de lodo ativado de uma indústria têxtil. Testes foram realizados a temperatura ambiente, empregando-se $5 \mathrm{mg} / \mathrm{L}$ de corante e $5 \mathrm{~g} / \mathrm{L}$ de adsorvente. Primeiramente avaliouse a influência do $\mathrm{pH}$, onde constatou-se que ambos os adsorventes apresentaram melhor resultado com o $\mathrm{pH} \mathrm{7.} \mathrm{O} \mathrm{carvão} \mathrm{obtido} \mathrm{a} \mathrm{partir} \mathrm{do} \mathrm{lodo} \mathrm{demonstrou}$ melhor eficiência de adsorção (89\% de remoção do corante em 16 h) e foi empregado para o estudo cinético. Observou-se que a cinética de pseudo segunda ordem apresentou-se mais adequada.
\end{abstract}

\section{INTRODUÇÃO}

A indústria têxtil é um setor que gera elevados níveis de poluição. Este ramo industrial tem grande participação no que diz respeito à poluição dos recursos hídricos, uma vez que a água é um insumo largamente utilizado em seus processos fabris. Por meio de legislações e resoluções, toda indústria deve tratar seu efluente antes de devolvê-lo ao meio seguindo determinados padrões governamentais.

Existem diversas formas de tratar efluentes, dentre os quais, métodos físicos e químicos que são utilizados para remover as impurezas após as etapas de fabricação dos produtos. Floculação, coagulação, tanques aerados, adsorção, entre outros, são os mais utilizados. Algumas dessas técnicas de tratamento têm se mostrado eficientes, embora possuam limitações (Fu e Viraraghevan, 2000; Dallago, Smaniotto e Oliveira, 2005; Aksu, 2001). Apesar de alguns suprirem muito bem a remoção dos sólidos dissolvidos, muitas vezes deixam a desejar quando se diz respeito à cor. Dentre as várias opções de tratamento, a adsorção se mostra bastante satisfatória para a remoção deste parâmetro em efluentes. Como citado por Cardoso et al. (2011), vários fatores interferem no desempenho do processo tais como textura da superfície do adsorvente, $\mathrm{pH}$ e tempo de contato.

Por este motivo, diversos produtos já foram testados como adsorventes. Além de carvões ativos comerciais, podem ser citados os produtos residuais agrícolas, como bagaço de 


\section{Congresso Brasileiro de Engenharia Química em Iniciação Científica Unicamp - Campinas - SP 19 a 22 de julho de 2015}

cana e casca de frutos; ou ainda os minerais, como terra diatomácea, óxidos metálicos, resíduos de fundição, entre outros (Ye et al., 2006; Ahmad e Alrozi, 2010). Além desses materiais agrícolas, materiais residuais têm se tornado um objeto de considerável interesse, tais como o lodo resultante da produção do biogás nos biodigestores anaeróbios, quitosana (Chiou e Li, 2003; Wu, Tseng e Juang, 2001) e lodo seco proveniente de alguns ramos da indústria após o processo de tratamento de efluentes (Vasques et al., 2011).

Com base no exposto, este trabalho teve como objetivo comparar a eficiência de remoção do corante reativo Preto 5 com um carvão comercial de casca de coco e com um carvão obtido a partir de lodo ativado gaseificado. Para tanto, primeiramente foi avaliado o melhor $\mathrm{pH}$ com os dois adsorventes e em seguida, a cinética do processo empregando o carvão que apresentou melhor eficiência de adsorção.

\section{MATERIAIS E MÉTODOS}

Nos testes avaliados empregou-se temperatura ambiente e os ensaios foram realizados em shaker para frascos de erlenmeyer de $250 \mathrm{~mL}$ empregando $100 \mathrm{~mL}$ de volume útil com solução sintética de corante, carvão e água destilada.

\subsection{Materiais}

Os adsorventes empregados para a realização do experimento foram: carvão ativado comercial de casca de coco Carbomafra, granulometria 1,05 - 1,30 mm fornecido pela Indústria Química Carbomafra S.A e lodo seco provindo da estação de tratamento de efluente de uma indústria têxtil de Blumenau - SC, gaseificado em mufla a $550{ }^{\circ} \mathrm{C}$. O corante empregado foi preto reativo Remazol N Gran 150\% C.I Black 5 da empresa Dystar. Soluções de ácido acético e de hidróxido de sódio foram empregadas para correção do $\mathrm{pH}$ (ajuste realizado com auxílio de um pHmetro).

\subsection{Metodologia}

Comparação entre adsorventes: Os ensaios de adsorção visando a comparação entre adsorventes foram executados empregando-se $\mathrm{pH}$ inicial nos valores 5,7 e 9 . A concentração de corante utilizado foi de $5 \mathrm{mg} / \mathrm{L}$ e de adsorvente foi $5 \mathrm{~g} / \mathrm{L}$. Os frascos foram deixados sob agitação de $116 \mathrm{rpm}$ por um período de 16 horas em um shaker. A determinação da concentração residual de corante no banho foi realizada por meio de absorbância, onde para tal método fez-se uso de uma curva de calibração. Para tanto, foi utilizado um espectrofotômetro analisando-se as amostras com comprimento de onda ajustado para 598 nm.

Ensaios para estudo cinético da adsorção do corante preto: A partir dos resultados obtidos na etapa anterior, seguiu-se com o estudo cinético empregando-se apenas o adsorvente que apresentou melhor resultado. Os ensaios foram realizados com a mesma concentração de corante e adsorvente. Amostras das fases líquidas foram retiradas e analisadas por espectrofotometria do tempo inicial em 10, 20, 30, 60, 90, 120 e 180 minutos. A partir destes resultados foi construída a cinética da concentração residual de corante no banho. 


\section{Congresso Brasileiro de Engenharia Química em Iniciação Científica Unicamp - Campinas - SP 19 a 22 de julho de 2015}

A avaliação da capacidade de adsorção do carvão foi realizada empregando-se as equações (1) e (2) que representam respectivamente a concentração de corante no adsorvente e a eficiência de remoção.

$$
\begin{aligned}
& q e=[(\mathrm{Co}-\mathrm{Ce}) \times V] / m \\
& R \%=[(\mathrm{Co}-\mathrm{Cf}) / \mathrm{Co}] \times 100
\end{aligned}
$$

\section{RESULTADOS E DISCUSSÕES}

\subsection{Avaliação de eficiência de remoção com diferentes adsorventes.}

Os resultados obtidos de adsorção em testes de $16 \mathrm{~h}$ com diferentes valores de $\mathrm{pH}$ comparando-se o carvão comercial de casca de coco e o lodo ativado gaseificado são apresentados na Tabela 1. Observa-se que os maiores valores na remoção de corante foram obtidos em $\mathrm{pH} \mathrm{7,} \mathrm{independente} \mathrm{do} \mathrm{adsorvente} \mathrm{utilizado.} \mathrm{Conforme} \mathrm{citado} \mathrm{por} \mathrm{Orfão} \mathrm{et} \mathrm{al.}$ (2006), o pH da solução exerce um papel importante no processo uma vez que influencia na carga da superfície do adsorvente e consequentemente na intensidade das interações eletrostáticas entre a superfície e as moléculas de corante. Ressalta-se que não foi observado uma tendência crescente (ou decrescente) com o aumento ou diminuição do valor do $\mathrm{pH}$, mas apenas o melhor resultado com $\mathrm{pH}$ 7. Este resultado é diferente do relatado por Aljeboree et al. (2014) que observou uma tendência crescente de adsorção com o decréscimo do pH utilizando carvão ativado de casca de coco. No entanto, os autores trabalharam com corantes diferentes e valores de $\mathrm{pH}$ mais extremos. Dessa forma, recomenda-se um estudo mais aprofundado para avaliação da melhor condição a ser empregada para esta variável.

Tabela 1 - Resultados de adsorção com os adsorventes testados em diferentes valores de $\mathrm{pH}$.

\begin{tabular}{ccccccc}
\hline Carvão & \multicolumn{3}{c}{ Carvão de casca de coco } & \multicolumn{3}{c}{ Carvão de lodo ativado gaseificado } \\
\hline \multirow{2}{*}{$\mathrm{pH}$} & $\begin{array}{c}\text { Conc. } \\
\text { Inicial } \\
(\mathrm{mg} / \mathrm{L})\end{array}$ & $\begin{array}{c}\text { Conc. } \\
\text { Final } \\
(\mathrm{mg} / \mathrm{L})\end{array}$ & $\begin{array}{c}\text { Remoção } \\
(\%)\end{array}$ & $\begin{array}{c}\text { Conc. } \\
\text { Inicial } \\
(\mathrm{mg} / \mathrm{L})\end{array}$ & $\begin{array}{c}\text { Conc. } \\
\text { Final } \\
(\mathrm{mg} / \mathrm{L})\end{array}$ & $\begin{array}{c}\text { Remoção } \\
(\%)\end{array}$ \\
\hline 5 & 5 & 2,49 & 50,26 & 5 & 0,83 & 83,42 \\
7 & 5 & 1,92 & 61,66 & 5 & 0,52 & 89,64 \\
9 & 5 & 2,12 & 57,51 & 5 & 1,09 & 78,24 \\
\hline
\end{tabular}

Também, foi constatado que o carvão gaseificado a $550{ }^{\circ} \mathrm{C}$ apresentou melhores resultados em relação ao carvão ativado de casca de coco. Este comportamento também era esperado. De fato, tratamentos térmicos e químicos maximizam a capacidade de adsorção uma vez que a superfície do adsorvente é alterada (Orfão et al., 2006). Assim, a partir destes resultados, o carvão selecionado para realização do estudo da cinética de adsorção do corante preto reativo 5 foi o lodo gaseificado $\mathrm{em} \mathrm{pH} 7$.

\subsection{Estudo Cinético}

Os resultados da cinética de adsorção com o carvão de lodo gaseificado são apresentados na Figura 1(a) para a concentração de corante residual na solução, e na Figura 


\section{Congresso Brasileiro de Engenharia Química em Iniciação Científica Unicamp - Campinas - SP 19 a 22 de julho de 2015}

1(b) para a concentração de corante no adsorvente. Observa-se que à medida que a concentração de corante na solução vai diminuindo em função do tempo, a mesma vai aumentando no interior do adsorvente, conforme esperado. O comportamento apresentado pode ser comparado aos resultados de Ahmad et al. (2014) realizados também com adsorção de corante reativo e que demonstraram grande quantidade de corante adsorvido no período inicial (2h), fato este que se deve a maior disponibilidade de poros vazios na superfície do carvão ativado. A reação se tornou mais lenta até se atingir o equilíbrio, uma vez que havia menor quantidade de orifícios vazios. Nos experimentos realizados neste estudo, o período de $2 \mathrm{~h}$ correspondeu ao total avaliado e observa-se que neste tempo a reação estava alcançando o equilíbrio.

Figura 1 - Cinética de adsorção do corante preto reativo 5 na solução (a) e no adsorvente (b) com carvão de lodo gaseificado em pH 7.

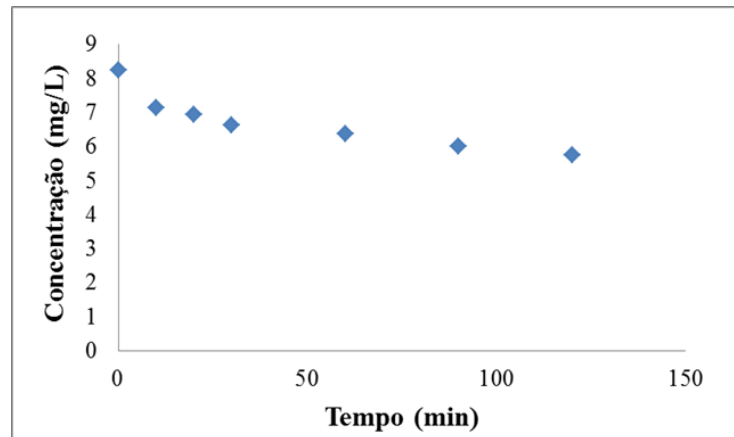

(a)

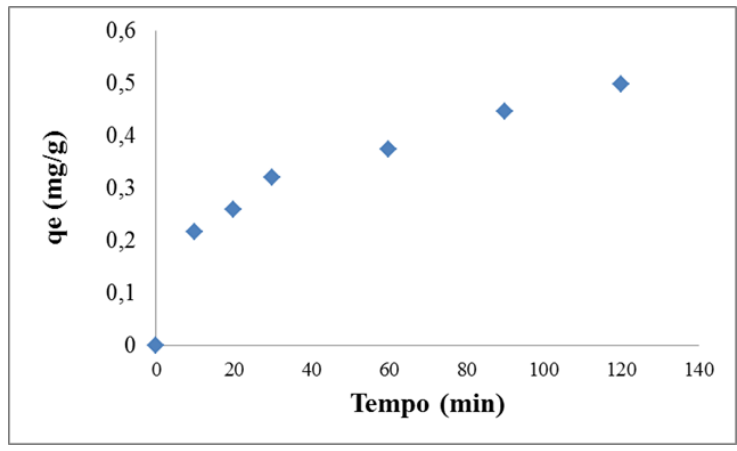

(b)

A comparação entre os modelos cinéticos de pseudo primeira e pseudo segunda ordem é apresentada na Figura 2 (dados linearizados). A partir dos valores das correlações obtidos nos ajustes realizados, é possível constatar que a cinética de pseudo segunda ordem é mais adequada ao processo em estudo. Observa-se que os dados tratados apresentam maior uniformidade na Figura 2(b) bem como a correlação que representa o ajuste realizado é melhor representada pela cinética de pseudo segunda ordem. Este resultado está de acordo com os obtidos por Ahmad et al. (2014) que também observaram melhor ajuste com cinética de pseudo segunda ordem em adsorção de corante reativo.

Figura 2 - Cinética de pseudo Primeira Ordem (a) e pseudo Segunda Ordem (b) para a remoção do corante preto reativo 5 com carvão de lodo gaseificado em pH 7.

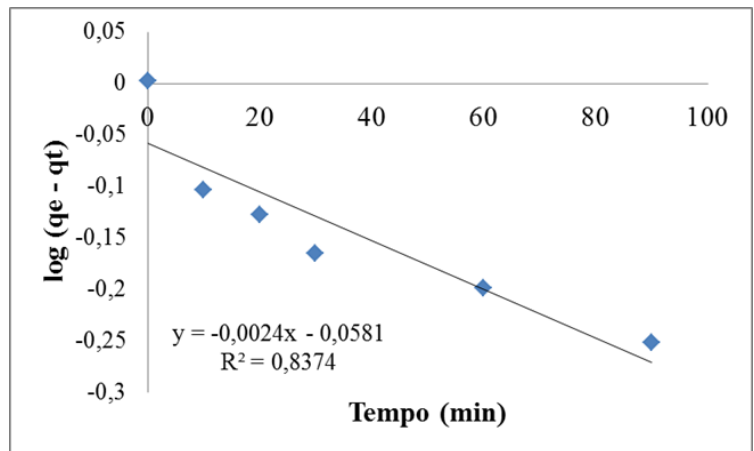

(a)

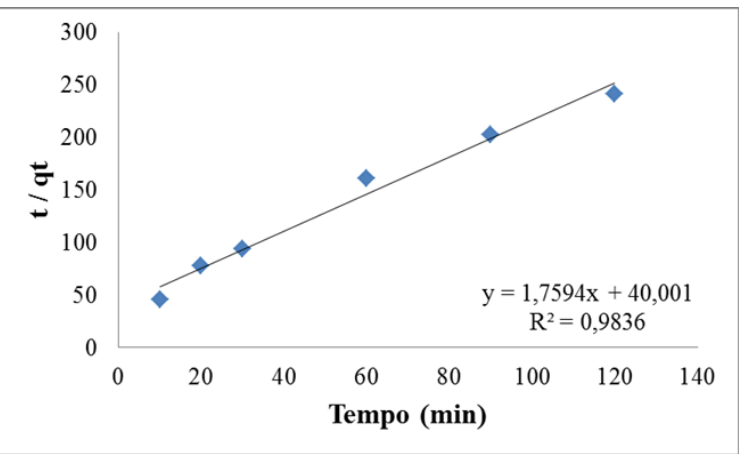

(b) 
A partir dos resultados apresentados na Figura 2(b), os valores da concentração de corante no adsorvente $\left(\mathrm{q}_{\mathrm{e}}\right)$ e da constante $\mathrm{k}$ foram calculados. Com estes valores o cálculo de $\mathrm{q}_{\mathrm{t}}$ foi realizado conforme a equação geral da cinética de pseudo segunda ordem (3), obtendose então dados modelados. A comparação destes com os obtidos experimentalmente é visualizada na Figura 3. O valor obtido para a constante de pseudo segunda ordem foi de $0,077 \mathrm{~g} / \mathrm{mg} \mathrm{min}$.

$$
q t=\left(k_{s} q_{e}^{2} t\right) /\left(1+q_{e} k_{s} t\right)
$$

Figura 3 - Comparação da cinética obtida a partir dos dados experimentais e modelados.

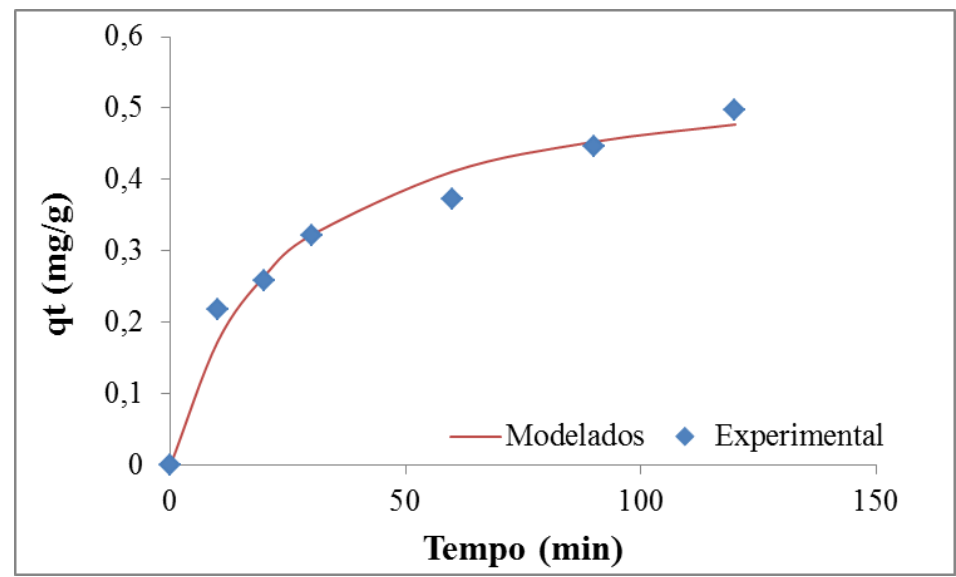

A visualização da Figura 3 permite verificar que os dados modelados representam adequadamente os dados experimentais.

\section{CONCLUSÃO}

Neste trabalho foi demonstrado que a utilização de um carvão obtido pela gaseificação de lodo de estação de tratamento de efluentes apresentou melhor eficiência de adsorção com corante reativo preto 5 em relação a um carvão comercial de casca de coco. A cinética de pseudo segunda ordem foi identificada como a mais representativa para o processo de adsorção para o carvão gaseificado.

Os resultados obtidos demonstram a viabilidade do uso do carvão de lodo gaseificado bem como apresentam dados em relação a este processo de adsorção que incentivam avançar em pesquisas mais detalhadas com este material, investigando diferentes condições de operação, bem como avaliar o processo com diferentes corantes.

\section{NOMENCLATURA}

$\mathrm{C}_{\mathrm{e}}=$ Concentração de equilíbrio de corante na solução (mg/L)

$\mathrm{C}_{\mathrm{f}}=$ Concentração final de corante na solução $(\mathrm{mg} / \mathrm{L})$

$\mathrm{C}_{\mathrm{o}}=$ Concentração inicial de corante na solução $(\mathrm{mg} / \mathrm{L})$

$\mathrm{k}_{\mathrm{s}}=$ Constante de pseudo segunda ordem $\left(\mathrm{h}^{-1}\right)$

$\mathrm{q}_{\mathrm{e}}=$ Concentração de corante no adsorvente no equilíbrio $(\mathrm{mg} / \mathrm{g})$ 


\section{Congresso Brasileiro de Engenharia \\ Química em Iniciação Científica Unicamp - Campinas - SP \\ 19 a 22 de julho de 2015}

$\mathrm{q}_{\mathrm{t}}=$ Concentração de corante no adsorvente no tempo $\mathrm{t}(\mathrm{mg} / \mathrm{g})$

$\mathrm{R}=$ Eficiência de remoção de corante da solução (\%)

\section{REFERENCIAS}

AHMAD, M. A.; ALROZI, R. Optimization of preparation conditions for mangosteen peelbased activated carbons for the removal of Remazol Brilliant Blue R using response surface methodology, Chem. Eng. J., v. 165, p. 883 - 890, 2010.

AHMAD, M. A; HERAWAN, S. G.; YUSOF, A. A. Equilibrium, Kinetics, and Thermodynamics of Remazol Brilliant Blue R Dye Adsorption onto Activated Carbon Prepared from Pinang Frond, ISRN Mech. Eng., v. 2014, p. 1 - 7, 2014.

AKSU, Z. Biosorption of reactive dyes by dried activated sludge: equilibrium and kinetic modeling. Biochem. Eng. J., v. 7, p. 79-84, 2001.

ALJEBOREE, A.M., ALSHIRIFI, A. N., ALKAIM, A. F., Kinetics and equilibrium study for the adsorption of textile dyes on coconut shell activated carbon. Arabian Journal of Chemistry, doi:10.1016/j.arabjc.2014.01.020, 2014.

CARDOSO, N. F; PINTO, R. B; LIMA, E. D; CALVETE, T.; AMAVISCA, C. V, ROYER, B.; CUNHA, M. L.; FERNANDES T. H. H.; PINTO, I. S. Remozal of remazol black B textile dye from aqueous solution by adsorption. Desalination, v. 269, p. 92-103, 2011.

CHIOU, M. S.; LI, H. Y., Adsorption behavior of reactive dye in aqueous solution on chemical cross-linked chitosan beads. Chemosphere, v. 50, p. 1095-1105, 2003.

DALlAGO, R. M.; SMANIOTTO, A.; OLIVEIRA, L. C. A. Resíduos sólidos de curtumes como adsorventes para a remoção de corantes em meio aquoso. Quím. Nova, v. 28, p. 433-437, 2005.

FU, Y. Z.; VIRARAGHAVAN, T. Removal of a dye from an aqueous solution by the fungus Aspergillus niger. Water Qual. Res. J. Can., v. 35, p. 95-111, 2000.

ÓRFÃO, J. J. M.; SILVA A. I. M.; PEREIRA J. C. V.; BARATA S. A.; FONSECA I. M.; FARIA P. C. C.; PEREIRA M. F. R. Adsorption of a reactive dye on chemically modified activated carbons - Influence of pH. J. Colloid and Inteface Sci., v. 296, p. 480-489, 2006.

VASQUES, A. R.; de SOUZA, S. M. A. G. U.; WEISSENBERG, L.; de SOUZA, A. A. U.; VALLE, J. A. B. Adsorção dos corantes RO16, RR2 e RR141 utilizando lodo residual da indústria têxtil. Eng. Sanit. Ambient, v. 16, p. 245-252, 2011.

WU, F. C.; TSENG, R. L.; JUANG, R. S. Kinetic modeling of liquid-phase adsorption of reactive dyes and metal ions on chitosan. Water Res., v. 35, p. 613-618, 2001.

YE, H.; CHEN, F.; SHENG, Y.; SHENG, G.; FU, J. Adsorption of phosphate from aqueous solution onto modified palygorskites. Sep. Purif. Technol., v. 50, p. 283 - 290, 2006. 